4 McAllister HA, Fenoglio JJ. Cardiac involvement in Whipple's disease. Circulation 1975;52:152-6

5 Bostwick DG, Bensch KG, Burke JS, et al. Whipple's disease presenting as aortic insufficiency. $N$ Engl J Med 1981;305:995-8

6 Elkins C, Todd A, Shuman MD, Pirolo JS. Cardiac Whipple's disease without digestive symptoms. Ann Thorac Surg 1999;67:250-1

7 Grijalva M, Horvath R, Dendis M, Cerny J, Benedik J. Molecular diagnosis of culture negative endocarditis: clinical validation in a group of surgically treated patients. Heart 2003;89:263-8

\section{Hepatic epithelioid haemangioendothelioma}

\author{
Elizabeth Haydon BSc ${ }^{1}$ Geoffrey Haydon MD MRCP ${ }^{2}$ \\ Simon Bramhall MD FRCS ${ }^{2}$ A David Mayer MS FRCS ${ }^{2}$ \\ Desley Niel MB MRCPath ${ }^{3}$
}

J R Soc Med 2005;98:364-365

CLINICAL SECTION, 19 FEBRUARY 2004

Primary liver tumours have a varied prognosis. Orthotopic liver transplantation has the potential to cure some of these cancers, and these cases need to be identified.

\section{CASE HISTORY}

A woman of 47 reported a dull ache in her right shoulder which, over the next few months, worsened and moved to her abdomen; it was associated with epigastric fullness, nausea, anorexia and tiredness. Fifteen months from symptom onset she had lost $5 \mathrm{~kg}$ and was experiencing a grabbing right upper quadrant and epigastric pain on deep breathing and coughing. She was unable to lie flat because of the pain. Initially, dietary treatment for gallstones slightly lessened the symptoms, but at twenty-three months the pain was incapacitating - severe, though fluctuating, and now extending to the left upper quadrant. She was admitted for investigation.

On examination there were no stigmata of chronic liver disease, masses or organomegaly. Ultrasound showed no abnormalities, and ultrasound-guided liver biopsy at this stage was inconclusive. Her alcohol history was 1-2 units per week. The differential diagnosis included acute-onchronic cholecystitis, peptic ulceration and recurrent acute pancreatitis. A second ultrasound-guided liver biopsy then showed hepatic epithelioid haemangioendothelioma (Figure 1). CT revealed several focal lesions, each up to $3 \mathrm{~cm}$ in diameter. She was referred to the regional liver unit, where orthotopic liver transplantation was performed nine months later. Figure 2 shows a slice of the explanted

${ }^{1}$ Medical School, University of Birmingham; ${ }^{2}$ Liver Unit and ${ }^{3}$ Department of Pathology, Queen Elizabeth Hospital, Birmingham B15 2TH, UK

Correspondence to: Mr Simon Bramhall FRCS liver, with several discrete and coalescing pale lesions. Serum aspartate aminotransferase was normal by day 8 and the patient returned to work after ten weeks. Sixteen months postoperatively she was in good health, and on ultrasound scanning the liver was normal in shape and echotexture, with no focal lesions or biliary dilatation. There was no free fluid in the abdomen, the spleen and kidneys were of normal size and liver function tests were within the normal range.

\section{COMMENT}

Hepatic epithelioid haemangioendothelioma is a rare multifocal vascular tumour ${ }^{1}$ that can develop at any age, with a female preponderance. The aetiology is unclear, although vinyl chloride, liver trauma and exogenous hormones ${ }^{1}$ have come under suspicion. Survival of affected individuals has ranged from a few months to many years. On the spectrum of malignancy haemangioendothelioma lies between benign haemangioma and malignant angiosarcoma. ${ }^{1,2}$ There are several diagnostic difficulties. First, the presentation is non-specific; second, liver function tests may not become abnormal until the disease is advanced; third, ultrasound scans of the liver can be inconclusive; and, finally, CT may lead to a misdiagnosis of metastatic disease because of the tumour's multifocal nature. On CT, multiple angiogenic lesions, $1-3 \mathrm{~cm}$ in diameter, ${ }^{3}$ are a common feature. The peripherally placed lesions are hypodense in the centre and cause enhancement of contrast media at the

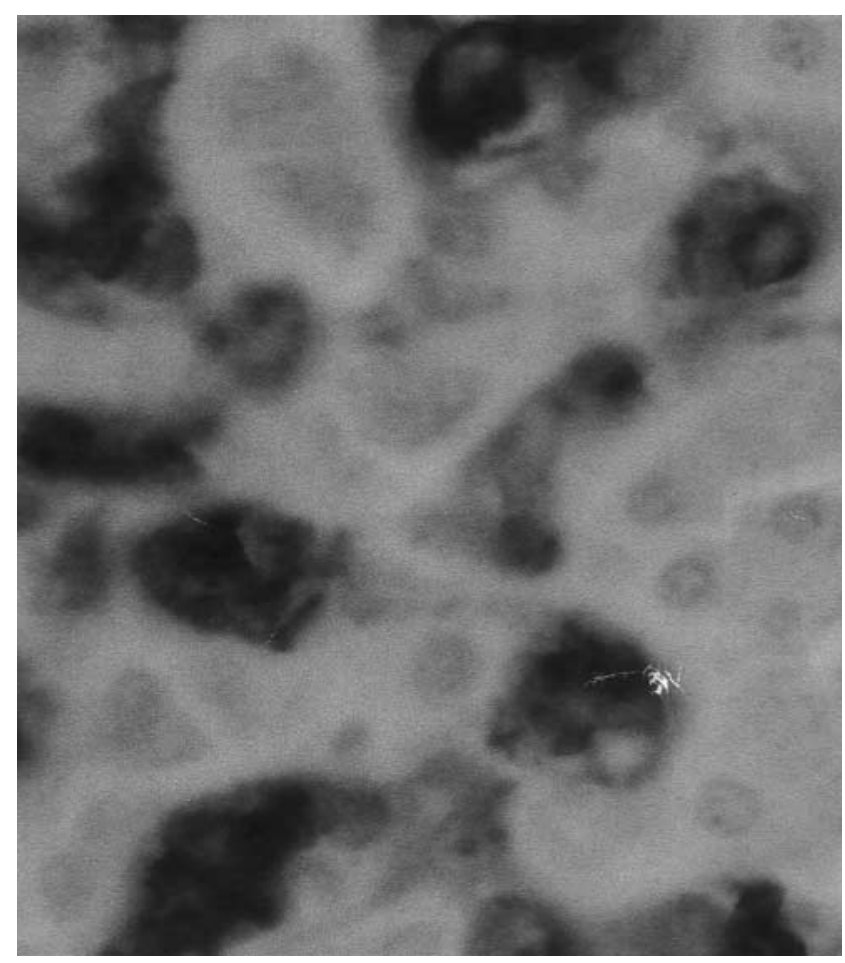

Figure 1 Sample of tumour obtained by ultrasound-guided liver biopsy. A proliferation of atypical endothelial cells is seen with CD31 immunostaining. Original magnification $\times 40$ 


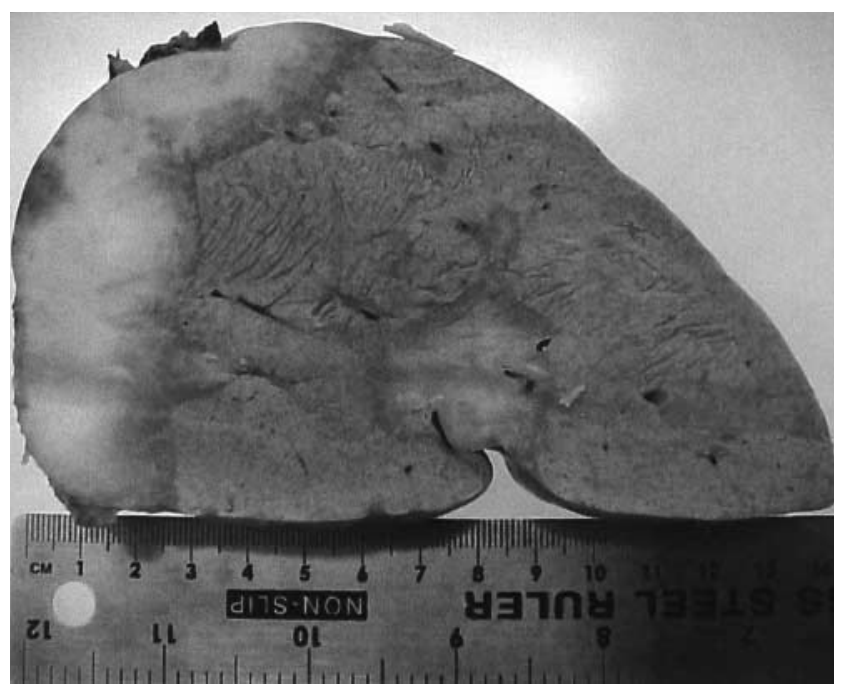

Figure 2 Slice of explanted liver showing multiple tumour nodules

periphery. In a patient with an abnormal liver ultrasound or CT, without a history of malignant disease or chronic liver disease, a guided liver biopsy may be appropriate. The biopsy appearance in the present patient was typical, with spindle-shaped tumour cells infiltrating the sinusoids and blood vessels. Intracytoplasmic vacuoles may be found, some containing red blood cells. ${ }^{3}$ Over time the tumours become sclerotic and calcified, and may coalesce to form a large fibrotic mass. Endothelial markers include factor VIII antigen, CD34 and CD31.

There are few treatment options. Chemotherapy and radiotherapy yield no improvement in survival, ${ }^{1}$ and surgical excision is usually impossible because of the multifocal nature of the disease; indeed, attempted resection may provoke aggressive regrowth. Orthotopic liver transplantation offers a possibility of long-term cure, ${ }^{5}$ although some patients do well without any treatment. How these 'indolent' cases can be identified is unknown. Experience of transplantation for this tumour at the Liver Transplant Unit in Birmingham is as follows. The first patient, transplanted in 1984, lived for 7 years; she died of lymphoproliferative disease. The second patient, transplanted in 1996, has recently been shown to have lung metastases; there is uncertainty whether metastases affect overall survival, and so far no treatment has been given. Three patients transplanted in 2003 are alive at the time of writing. Out of nearly 40000 liver transplants undertaken in Europe up to 2001, only $11 \%$ were for cancers $^{6}$ and 66 of these were for epithelioid haemangioendothelioma. 5 -year survival is estimated at $55-71 \% .^{7}$

\section{REFERENCES}

1 Mahklouf HR, Ishak KG, Goodman ZD. Epithelioid hemangioendothelioma. A clinicopathologic study of 137 cases. Cancer 1999;85:562-82
2 Lauffer JM, Zimmermann A, Krahenbuhl L, Triller J, Baer HU. Epithelioid hemangioma of the liver. A rare hepatic tumour. Cancer 1996; 78:2318-28

3 Elias KR, Ryan CK. Epithelioid hemangioma and the elusive vacuole. Liver Transplantation 2003;9:310-12

4 Schwartz ME. Hepatic epithelioid hemangioendothelioma: resection or transplantation, which and when? Liver Transplantation 1999;5:526-31

5 Nissen NN, Cavazzoni E, Tran TT, Poordad FP. Emerging role of transplantation for primary liver cancers. Cancer 2004;1:88-96

6 European Liver Transplant Registry [www.eltr.org]

7 Madariaga JR, Marino IR, Karavias DD, et al. Long term results after liver transplantation for primary hepatic epithelioid hemangioendothelioma. Ann Surg Oncol 1995;2:483-7

\section{Lymphatic fistula to the vagina}

\author{
S C Mills MRCS ${ }^{1}$ J Lee FRCS ${ }^{1}$ \\ M Marshall MRCP FRCS ${ }^{2}$ R Cohen FRCS ${ }^{1}$
}

J R Soc Med 2005;98:365-366

Lymphoceles and lymphatic fistulae are rare complications of abdominal surgery. External drainage of chyle via the rectum or vagina is unusual and difficult to manage.

\section{CASE HISTORY}

After panproctocolectomy for ulcerative colitis a woman of 44 developed a profuse watery vaginal discharge. There were no associated urinary tract or bowel symptoms and swabs from the discharge yielded no evidence of infection. CT revealed a substantial amount of intra-abdominal fluid, which was then drained by abdominoparacentesis, the drain being left in situ. On removal of the drain the discharge persisted. Again no microorganisms could be cultured from it. The watery nature of the discharge and a slight rightsided hydronephrosis noted on a repeat CT scan led to a full urological investigation for a presumptive ureteric or vesicovaginal fistula but none was found. At this point the clinicians speculated that the intra-abdominal fluid collection might be a lymphocyst draining into the vagina. A lymphangiogram was reported as normal but the adequacy of the study was questioned. A second lymphangiogram, deemed satisfactory, was reported as showing no evidence of a lymphatic leak. An exploratory laparotomy revealed no cause for the discharge and a hysterectomy and bilateral salpingo-oophorectomy were undertaken. The discharge

Departments of ${ }^{1}$ Colorectal Surgery and ${ }^{2}$ Radiology, St Mark's Hospital, Northwick Park, Watford Road, Harrow HA1 3UJ, Middlesex, UK

Correspondence to: Miss Sarah Mills

E-mail: sarah.mills@doctors.org.uk 